\title{
Note on the projectable linear connection in the semi-tangent bundle
}

\author{
Furkan Yildirim \\ Narman Vocational Training School, Ataturk University, 25530, Erzurum, Turkey \\ Received: 3 October 2021, Accepted: 26 November 2021 \\ Published online: 23 December 2021.
}

\begin{abstract}
The present paper is devoted to some results concerning with the projectable linear connection in the semi-tangent (pullback) bundle tM. In this study, horizontal lift problems of projectable linear connection, which are preliminary to the subject of covarient derivates of almost contact structure and almost paracontact structure on semi-tangent bundle, are discussed.
\end{abstract}

Keywords: Horizontal lift, Projectable linear connection, Pull-back bundle, Semi-tangent bundle, Vector field.

\section{Introduction}

Let $M_{n}$ be a differentiable manifold of class $C^{\infty}$ and finite dimension $n$, and let $\left(M_{n}, \pi_{1}, B_{m}\right)$ be a differentiable bundle over $B_{m}$. We use the notation $\left(x^{i}\right)=\left(x^{a}, x^{\alpha}\right)$, where the indices $i, j, \ldots$ run from 1 to $n$, the indices $a, b, \ldots$ from 1 to $n-m$ and the indices $\alpha, \beta, \ldots$ from $n-m+1$ to $n, x^{\alpha}$ are coordinates in $B_{m}, x^{a}$ are fibre coordinates of the bundle

$$
\pi_{1}: M_{n} \rightarrow B_{m}
$$

Let now $\left(T\left(B_{m}\right), \widetilde{\pi}, B_{m}\right)$ be a tangent bundle [13] over base space $B_{m}$, and let $M_{n}$ be differentiable bundle determined by a natural projection (submersion) $\pi_{1}: M_{n} \rightarrow B_{m}$. The semi-tangent bundle (pull-back [[2],[3],[9], [10],[14],[15]]) of the tangent bundle $\left(T\left(B_{m}\right), \widetilde{\pi}, B_{m}\right)$ is the bundle $\left(t\left(B_{m}\right), \pi_{2}, M_{n}\right)$ over differentiable bundle $M_{n}$ with a total space

$t\left(B_{m}\right)=\left\{\left(\left(x^{a}, x^{\alpha}\right), x^{\bar{\alpha}}\right) \in M_{n} \times T_{x}\left(B_{m}\right): \pi_{1}\left(x^{a}, x^{\alpha}\right)=\tilde{\pi}\left(x^{\alpha}, x^{\bar{\alpha}}\right)=\left(x^{\alpha}\right)\right\} \subset M_{n} \times T_{x}\left(B_{m}\right)$

and with the projection map $\pi_{2}: t\left(B_{m}\right) \rightarrow M_{n}$ defined by $\pi_{2}\left(x^{a}, x^{\alpha}, x^{\bar{\alpha}}\right)=\left(x^{a}, x^{\alpha}\right)$, where $T_{x}\left(B_{m}\right)\left(x=\pi_{1}(\widetilde{x}), \widetilde{x}=\left(x^{a}, x^{\alpha}\right) \in M_{n}\right)$ is the tangent space at a point $x$ of $B_{m}$, where $x^{\bar{\alpha}}=y^{\alpha}(\bar{\alpha}, \bar{\beta}, \ldots=n+1, \ldots, 2 n)$ are fibre coordinates of the tangent bundle $T\left(B_{m}\right)$.

Where the pull-back (Pontryagin [7]) bundle $t\left(B_{m}\right)$ of the differentiable bundle $M_{n}$ also has the natural bundle structure over $B_{m}$, its bundle projection $\pi: t\left(B_{m}\right) \rightarrow B_{m}$ being defined by $\pi:\left(x^{a}, x^{\alpha}, x^{\bar{\alpha}}\right) \rightarrow\left(x^{\alpha}\right)$, and hence $\pi=\pi_{1} \circ \pi_{2}$. Thus $\left(t\left(B_{m}\right), \pi_{1} \circ \pi_{2}\right)$ is the composite bundle [[8], p.9] or step-like bundle [6]. Consequently, we notice the semi-tangent bundle $\left(t\left(B_{m}\right), \pi_{2}\right)$ is a pull-back bundle of the tangent bundle over $B_{m}$ by $\pi_{1}$ [9].

If $\left(x^{i^{\prime}}\right)=\left(x^{a^{\prime}}, x^{\alpha^{\prime}}\right)$ is another local adapted coordinates in differentiable bundle $M_{n}$, then we have

$$
\left\{\begin{array}{l}
x^{a^{\prime}}=x^{a^{\prime}}\left(x^{b}, x^{\beta}\right), \\
x^{\alpha^{\prime}}=x^{\alpha^{\prime}}\left(x^{\beta}\right)
\end{array}\right.
$$

\footnotetext{
* Corresponding author e-mail: (F. Yıldırım) furkan.yildirim@ atauni.edu.tr
} 
The Jacobian of (1) has the components

$$
\left(A_{j}^{i^{\prime}}\right)=\left(\frac{\partial x^{i^{\prime}}}{\partial x^{j}}\right)=\left(\begin{array}{cc}
A_{b}^{a^{\prime}} & A_{\beta}^{a^{\prime}} \\
0 & A_{\beta}^{\alpha^{\prime}}
\end{array}\right),
$$

where $A_{b}^{a^{\prime}}=\frac{\partial x^{a^{\prime}}}{\partial x^{b}}, A_{\beta}^{a^{\prime}}=\frac{\partial x^{a^{\prime}}}{\partial x^{\beta}}, A_{\beta}^{\alpha^{\prime}}=\frac{\partial x^{\alpha^{\prime}}}{\partial x^{\beta}}[9]$

To a transformation (1) of local coordinates of $M_{n}$, there corresponds on $t\left(B_{m}\right)$ the change of coordinate

$$
\left\{\begin{array}{l}
x^{a^{\prime}}=x^{a^{\prime}}\left(x^{b}, x^{\beta}\right) \\
x^{\alpha^{\prime}}=x^{\alpha^{\prime}}\left(x^{\beta}\right) \\
x^{\bar{\alpha}^{\prime}}=\frac{\partial x^{\alpha^{\prime}}}{\partial x^{\beta}} y^{\beta}
\end{array}\right.
$$

The Jacobian of (2) is:

$$
\bar{A}=\left(A_{J}^{I^{\prime}}\right)=\left(\begin{array}{ccc}
A_{b}^{a^{\prime}} & A_{\beta}^{a^{\prime}} & 0 \\
0 & A_{\beta}^{\alpha^{\prime}} & 0 \\
0 & A_{\beta \varepsilon}^{\alpha^{\prime} y^{\varepsilon}} & A_{\beta}^{\alpha^{\prime}}
\end{array}\right)
$$

where $I=(a, \alpha, \bar{\alpha}), J=(b, \beta, \bar{\beta}), I, J, \ldots=1, \ldots, 2 n ; A_{\beta \varepsilon}^{\alpha^{\prime}}=\frac{\partial^{2} x^{\alpha^{\prime}}}{\partial x^{\beta} \partial x^{\varepsilon}}[9]$.

The purpose of this paper is to study the horizontal lifts of projectable linear connection to semi-tangent (pull-back) bundle $\left(t\left(B_{m}\right), \pi_{2}\right)$ and their properties.

We denote by $\mathfrak{I}_{q}^{p}\left(M_{n}\right)$ the set of all tensor fields of class $C^{\infty}$ and of type $(p, q)$ on $M_{n}$, i.e., contravariant degree $p$ and covariant degree $q$. We now put $\mathfrak{I}\left(M_{n}\right)=\sum_{p, q=0}^{\infty} \mathfrak{I}_{q}^{p}\left(M_{n}\right)$, which is the set of all tensor fields on $M_{n}$. Smilarly, we denote by $\mathfrak{I}_{q}^{p}\left(B_{m}\right)$ and $\mathfrak{I}\left(B_{m}\right)$ respectively the corresponding sets of tensor fields in the base space $B_{m}$.

\section{Some lifts of vector and covector fields}

If $f$ is a function on $B_{m}$, we write ${ }^{v v} f$ for the function on $t\left(B_{m}\right)$ obtained by forming the composition of $\pi: t\left(B_{m}\right) \rightarrow B_{m}$ and ${ }^{v} f=f \circ \pi_{1}$, so that

$$
{ }^{v v} f={ }^{v} f \circ \pi_{2}=f \circ \pi_{1} \circ \pi_{2}=f \circ \pi .
$$

Thus, the vertical lift ${ }^{v v} f$ of the function $f$ to $t\left(B_{m}\right)$ satisfies

$$
{ }^{v v} f\left(x^{a}, x^{\alpha}, x^{\bar{\alpha}}\right)=f\left(x^{\alpha}\right)
$$

We note here that value ${ }^{v v} f$ is constant along each fibre of $\pi: t\left(B_{m}\right) \rightarrow B_{m}$. Let $X \in \mathfrak{I}_{0}^{1}\left(B_{m}\right)$, i.e. $X=X^{\alpha} \partial_{\alpha}$. On putting

$$
{ }^{v v} X=\left({ }^{v v} X^{\alpha}\right)=\left(\begin{array}{l}
0 \\
0 \\
X^{\alpha}
\end{array}\right),
$$

from (3), we easily see that ${ }^{v v} X^{\prime}=\bar{A}\left({ }^{v v} X\right)$. The vector field ${ }^{v v} X$ is called the vertical lift of $X$ to $t\left(B_{m}\right)$. 
Let $\omega \in \mathfrak{I}_{1}^{0}\left(B_{m}\right)$, i.e. $\omega=\omega_{\alpha} d x^{\alpha}$. On putting

$$
{ }^{v v} \omega=\left({ }^{v v} \omega\right)_{\alpha}=\left(0, \omega_{\alpha}, 0\right),
$$

from (3), we easily see that ${ }^{v v} \omega=\bar{A}^{v v} \omega^{\prime}$. The covector field ${ }^{v v} \omega$ is called the vertical lift of $\omega$ to $t\left(B_{m}\right)$.

Let $\widetilde{X} \in \mathfrak{I}_{0}^{1}\left(M_{n}\right)$ be a projectable vector field [11] with projection $X=X^{\alpha}\left(x^{\alpha}\right) \partial_{\alpha}$ i.e. $\widetilde{X}=\widetilde{X}^{a}\left(x^{a}, x^{\alpha}\right) \partial_{a}+X^{\alpha}\left(x^{\alpha}\right) \partial_{\alpha}$. Now, consider $\widetilde{X} \in \mathfrak{I}_{0}^{1}\left(M_{n}\right)$, then ${ }^{c c} \widetilde{X}$ (complete lift) has the components on the semi-tangent bundle $t\left(B_{m}\right)$ [9]

$$
{ }^{c c} \widetilde{X}=\left({ }^{c} \widetilde{X}^{\alpha}\right)=\left(\begin{array}{l}
\widetilde{X}^{a} \\
X^{\alpha} \\
y^{\varepsilon} \partial_{\varepsilon} X^{\alpha}
\end{array}\right)
$$

with respect to the coordinates $\left(x^{a}, x^{\alpha}, x^{\bar{\alpha}}\right)$.

For any $F \in \mathfrak{I}_{1}^{1}\left(B_{m}\right)$, if we take account of (3), we can prove that $(\gamma F)^{\prime}=\bar{A}(\gamma F)$, where $\gamma F$ is a vector field defined by

$$
\gamma F=\left(\gamma F^{I}\right)=\left(\begin{array}{l}
0 \\
0 \\
y^{\varepsilon} F_{\varepsilon}^{\alpha}
\end{array}\right)
$$

with respect to the coordinates $\left(x^{a}, x^{\alpha}, x^{\bar{\alpha}}\right)$.

Let now $\widetilde{X} \in \mathfrak{I}_{0}^{1}\left(M_{n}\right)$ be a projectable vector field on $M_{n}$ with projection $X \in \mathfrak{I}_{0}^{1}\left(B_{m}\right)$ [11]. Then we define the horizontal lift ${ }^{H H} \widetilde{X}$ of $\widetilde{X}$ by

$$
{ }^{H H} \widetilde{X}={ }^{c} \widetilde{X}-\gamma(\nabla \widetilde{X})
$$

on $t\left(M_{n}\right)$. Where $\nabla$ is a projectable symmetric linear connection in a differentiable manifold $B_{m}$. Then, remembering that ${ }^{c} \widetilde{X}$ and $\gamma(\nabla \widetilde{X})$ have, respectively, local componenets

$$
{ }^{c c} \widetilde{X}=\left({ }^{c c} \widetilde{X}^{I}\right)=\left(\begin{array}{l}
\widetilde{X}^{a} \\
X^{\alpha} \\
y^{\varepsilon} \partial_{\varepsilon} X^{\alpha}
\end{array}\right), \gamma(\nabla \widetilde{X})=\left(\gamma(\nabla \widetilde{X})^{I}\right)=\left(\begin{array}{l}
0 \\
0 \\
y^{\varepsilon} \nabla_{\varepsilon} X^{\alpha}
\end{array}\right)
$$

with respect to the coordinates $\left(x^{a}, x^{\alpha}, x^{\bar{\alpha}}\right)$ on $t\left(B_{m}\right) . \nabla_{\alpha} X^{\varepsilon}$ being the covariant derivative of $X^{\varepsilon}$, i.e.,

$$
\left(\nabla_{\alpha} X^{\varepsilon}\right)=\partial_{\alpha} X^{\varepsilon}+X^{\beta} \Gamma_{\beta}^{\varepsilon}
$$

We find that the horizontal lift ${ }^{H H} \widetilde{X}$ of $\widetilde{X}$ has the components

$$
{ }^{H H} X=\left({ }^{H H} X^{I}\right)=\left(\begin{array}{l}
\widetilde{X}^{a} \\
X^{\alpha} \\
-\Gamma_{\beta}^{\alpha} X^{\beta}
\end{array}\right)
$$

with respect to the coordinates $\left(x^{a}, x^{\alpha}, x^{\bar{\alpha}}\right)$ on $t\left(B_{m}\right)$. Where

$$
\Gamma_{\beta}^{\alpha}=y^{\varepsilon} \Gamma_{\varepsilon \beta}^{\alpha} .
$$




\section{Complete lifts of projectable linear connection}

Let $\Gamma_{\alpha \gamma}^{\beta}$ be components of projectable linear connection [[1], [4], [5], [11], [12]] $\nabla$ with respect to local coordinates $\left(x^{\alpha}\right)$ in $B_{m}$ and ${ }^{c c} \Gamma_{I}^{J}{ }_{K}$ components of ${ }^{c c} \nabla$ with respect to the induced coordinates $\left(x^{a}, x^{\alpha}, x^{\bar{\alpha}}\right)$ in $t\left(B_{m}\right)$. We recall from [11] that components ${ }^{c c} \Gamma_{I}^{J}{ }_{K}$ of complete lift ${ }^{c c} \nabla$ of projectable linear connection $\nabla$ can be calculated from base manifold $B_{m}$ to semi-tangent bundle $t\left(B_{m}\right)$ also as:

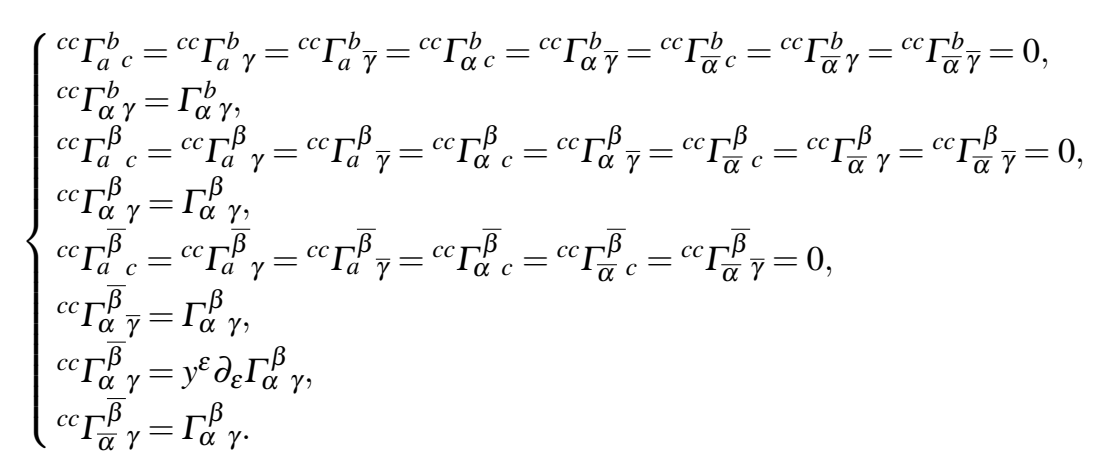

where $I=(a, \alpha, \bar{\alpha}), J=(b, \beta, \bar{\beta}), K=(c, \gamma, \bar{\gamma})$. On the other hand, from (11) we obtain:

Theorem 1. Let $\widetilde{X}$ and $\widetilde{Y}$ be projectable vector fields on $M_{n}$ with projection $X \in \mathfrak{I}_{0}^{1}\left(B_{m}\right)$ and $Y \in \mathfrak{I}_{0}^{1}\left(B_{m}\right)$, respectively. We have:

(i) ${ }^{c c} \nabla^{v_{X}}\left({ }^{v v} Y\right)=0$,

(ii) ${ }^{c c} \nabla_{v^{v}}\left({ }^{H H} \widetilde{Y}\right)=0$,

(iii) ${ }^{c c} \nabla_{H H \widetilde{X}}\left({ }^{v v} Y\right)={ }^{v v}\left(\nabla_{X} Y\right)$,

(iv) ${ }^{c c} \nabla_{H H \tilde{X}}\left({ }^{H H} \widetilde{Y}\right)={ }^{H H}\left(\nabla_{X} Y\right)+\gamma(R(, X) Y)$,

(v) $\left[{ }^{c c} \widetilde{X},{ }^{c c} \widetilde{Y}\right]={ }^{c c}[\widetilde{X}, \widetilde{Y}]\left(\right.$ i.e. $\left.L_{c c} \widetilde{X}\left({ }^{c c} \widetilde{Y}\right)={ }^{c c}\left(L_{\widetilde{X}} \widetilde{Y}\right)\right)$,

(vi) $\left[{ }^{c c} \widetilde{X}, \gamma F\right]=\gamma\left(L_{X} F\right)\left(F \in \mathfrak{I}_{1}^{1}\left(B_{m}\right)\right)$,

where $R(, X) Y \in \mathfrak{I}_{1}^{1}\left(B_{m}\right)$ is a tensor field of type of $(1,1)$ defined by $F(Z)=R(Z, X) Y$ for any $Z \in \mathfrak{I}_{0}^{1}\left(B_{m}\right)$ and $L_{X}$ is the operator of Lie derivation with respect to $X$.

\section{Horizontal lifts of projectable linear connection}

Let there be given a projectable linear connection $\nabla$ in $B_{m}$. We shall define the horizontal lift ${ }^{H H} \nabla$ of a projectable linear connection $\nabla$ in $B_{m}$ to $t\left(B_{m}\right)$ by the conditions:

(i) ${ }^{H H} \nabla^{{ }^{v v}} X\left({ }^{v v} Y\right)=0$,

(ii) ${ }^{H H} \nabla^{{ }^{v} X}\left({ }^{H H} \widetilde{Y}\right)=0$

(iii) ${ }^{H H} \nabla_{H H \widetilde{X}}\left({ }^{v v} Y\right)={ }^{v v}\left(\nabla_{X} Y\right)$,

$$
(i v)^{H H} \nabla_{H H \widetilde{X}}\left({ }^{H H} \widetilde{Y}\right)={ }^{H H}\left(\nabla_{X} Y\right),
$$

for any $\widetilde{X}, \widetilde{Y} \in \mathfrak{I}_{0}^{1}\left(M_{n}\right)$. Thus, if we put

$$
\widetilde{S}(\widetilde{X}, \widetilde{Y})={ }^{H H} \nabla_{\widetilde{X}} \widetilde{Y}-{ }^{c c} \nabla_{\widetilde{X}} \widetilde{Y}
$$

for any $\widetilde{X}, \widetilde{Y} \in \mathfrak{I}_{0}^{1}\left(M_{n}\right)$. Then, from (13) and Theorem 1, the tensor $\widetilde{S}$ of type $(1,2)$ in $t\left(B_{m}\right)$ satisfies the conditions

(i) $\widetilde{S}\left({ }^{v v} X,{ }^{v v} Y\right)=0$,

(ii) $\widetilde{S}\left({ }^{v v} X,{ }^{H H} \widetilde{Y}\right)=0$,

(iii) $\widetilde{S}\left({ }^{H H} \widetilde{X},{ }^{v v} Y\right)=0$, 


$$
\text { (iv) } \widetilde{S}\left({ }^{H H} \widetilde{X},{ }^{H H} \widetilde{Y}\right)=-\gamma(R(, X) Y),
$$

for any $\widetilde{X}, \widetilde{Y} \in \mathfrak{I}_{0}^{1}\left(M_{n}\right)$. Therefore $\widetilde{S}$ has the components $\widetilde{S}_{I K}^{J}$ such that

$$
\widetilde{S}_{\alpha \gamma}^{\bar{\beta}}=-y^{\varepsilon} R_{\varepsilon \alpha \gamma}^{\beta}
$$

all others being zero, with respect to the induced coordinates $\left(x^{b}, x^{\beta}, x^{\bar{\beta}}\right)$ in $t\left(B_{m}\right)$.

Since the components ${ }^{c c} \Gamma_{I}^{J}{ }_{K}$ of ${ }^{c c} \nabla$ are given by (11), it follows from (13) and (15) that the horizontal lift ${ }^{H H} \nabla$ of a projectable linear connection $\nabla$ has the components ${ }^{H H} \Gamma_{I K}^{J}$ such that

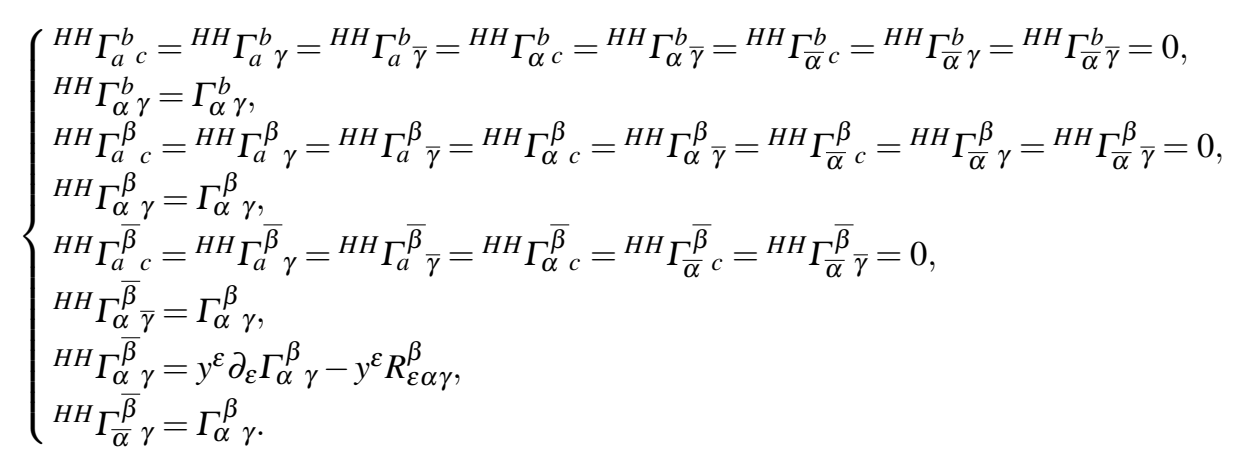

with respect to the induced coordinates in $t\left(B_{m}\right)$. Where ${ }^{H H} \Gamma_{I}^{J} K$ are the components of ${ }^{H H} \nabla$ in $t\left(B_{m}\right)$.

Proof. For convenience sake we only consider ${ }^{H}{ }^{\bar{\beta}} \Gamma_{\alpha}^{\bar{\beta}}$. According to (11), (13) and (15), in fact:

$$
\begin{gathered}
\widetilde{S}_{\alpha \gamma}^{\bar{\beta}}={ }^{H H} \Gamma_{\alpha \gamma}^{\bar{\beta}}-{ }^{c c} \Gamma_{\alpha \gamma}^{\bar{\beta}} \\
-y^{\varepsilon} R_{\varepsilon \alpha \gamma}={ }^{H H} \Gamma_{\alpha \gamma}^{\bar{\beta}}-y^{\varepsilon} \partial_{\varepsilon} \Gamma_{\alpha \gamma}^{\beta} \\
{ }^{H H} \Gamma_{\alpha \gamma}^{\bar{\beta}}=y^{\varepsilon} \partial_{\varepsilon} \Gamma_{\alpha \gamma}^{\beta}-y^{\varepsilon} R_{\varepsilon \alpha \gamma}^{\beta} .
\end{gathered}
$$

Thus, we have ${ }^{H H} \Gamma_{\alpha \gamma}^{\bar{\beta}}=y^{\varepsilon} \partial_{\varepsilon} \Gamma_{\alpha}^{\beta} \gamma-y^{\varepsilon} R_{\varepsilon \alpha \gamma}^{\beta}$. Similarly, we can easily find other components of ${ }^{H}{ }^{H} \Gamma_{I}^{J}$.

Theorem 2. Let $X, Y \in \mathfrak{I}_{0}^{1}\left(B_{m}\right)$. Then we obtain

$$
{ }^{H H} \nabla_{{ }^{v} X}\left({ }^{v v} Y\right)=0
$$

Proof. If $X, Y \in \mathfrak{I}_{0}^{1}\left(B_{m}\right)$ and

$$
\left(\begin{array}{c}
\left({ }^{H H} \nabla_{v^{v v}}\left({ }^{v v} Y\right)\right)^{b} \\
\left({ }^{H H} \nabla_{v^{v}}\left({ }^{v v} Y\right)\right)^{\beta} \\
\left({ }^{H H} \nabla_{v^{v}}\left({ }^{v v} Y\right)\right)^{\beta}
\end{array}\right)
$$

are the components of $\left({ }^{H H} \nabla_{v^{v} X}\left({ }^{(v} Y\right)\right)^{J}$ with respect to the coordinates $\left(x^{b}, x^{\beta}, x^{\bar{\beta}}\right)$ on $t\left(\boldsymbol{B}_{m}\right)$, then we have

$$
\left({ }^{H H} \nabla_{{ }^{v v} X}\left({ }^{v v} Y\right)\right)^{J}={ }^{v v} X^{a H H} \nabla_{a}\left({ }^{v v} Y\right)^{J}+{ }^{v v} X^{\alpha H H} \nabla_{\alpha}\left({ }^{v v} Y\right)^{J}+{ }^{v v} X^{\bar{\alpha} H H} \nabla_{\bar{\alpha}}\left({ }^{v v} Y\right)^{J} .
$$


Firstly, if $J=b$, we have

$$
\begin{aligned}
& \left({ }^{H H} \nabla_{{ }^{v}{ }_{X}}\left({ }^{v v} Y\right)\right)^{b}={ }^{v v} X^{a H H} \nabla_{a} \underbrace{v v}_{0} Y^{b}+{ }^{v v} X^{\alpha H H} \nabla_{\alpha} \underbrace{v^{v} Y^{b}}_{0}+{ }^{v v} X^{\bar{\alpha} H H} \nabla_{\bar{\alpha}} \underbrace{\left({ }^{v v} Y^{b}\right)}_{0} \\
& =0
\end{aligned}
$$

by virtue of (5) and (16). Secondly, if $J=\beta$, we have

$$
\begin{aligned}
\left({ }^{H H} \nabla^{{ }^{v v} X}\left({ }^{v v} Y\right)\right) & ={ }^{v v} X^{a H H} \nabla_{a} \underbrace{{ }^{v v} Y^{\beta}}_{0}+{ }^{v v} X^{\alpha H H} \nabla_{\alpha} \underbrace{{ }^{v v} Y^{\beta}}_{0}+{ }^{v v} X^{\bar{\alpha} H H} \nabla_{\bar{\alpha}} \underbrace{\left({ }^{v v} Y^{\beta}\right)}_{0} \\
& =0
\end{aligned}
$$

by virtue of (5) and (16). Thirdly, if $J=\bar{\beta}$, then we have

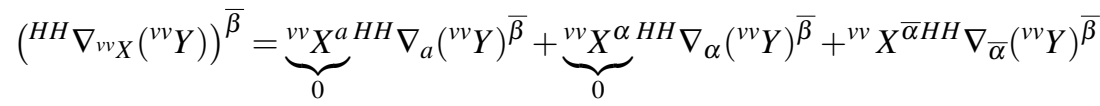

$$
\begin{aligned}
& =X^{\alpha}(\underbrace{\partial_{\alpha} Y^{\beta}}_{0}+{ }^{H H} \Gamma_{\bar{\alpha}}^{\beta} \underbrace{{ }^{v v} Y^{c}}_{0}+{ }^{H H} \Gamma_{\bar{\alpha}}^{\beta} \gamma \underbrace{\left({ }^{v v} Y\right)^{\gamma}}_{0}+\underbrace{{ }^{H H} \Gamma_{\bar{\alpha}}^{\beta} \bar{\gamma}}_{0}\left({ }^{v v} Y\right)^{\bar{\gamma}}) \\
& =0
\end{aligned}
$$

by virtue of (5) and (16). Thus Theorem 2 is proved.

Theorem 3. Let $\widetilde{Y}$ be a projectable vector field on $M_{n}$ with projections $Y$ on $B_{m}$. If $X \in \mathfrak{I}_{0}^{1}\left(B_{m}\right)$, then

$$
{ }^{H H} \nabla_{{ }^{v} X}\left({ }^{H H} \widetilde{Y}\right)=0 .
$$

Proof. If $\widetilde{Y} \in \mathfrak{I}_{0}^{1}\left(M_{n}\right), X \in \mathfrak{I}_{0}^{1}\left(B_{m}\right)$ and

$$
\left(\begin{array}{c}
\left({ }^{H H} \nabla^{v v} X\right. \\
\left.\left({ }^{H H} \widetilde{Y}\right)\right)^{b} \\
\left({ }^{H H} \nabla_{v^{v} X}\left({ }^{H H} \widetilde{Y}\right)\right)^{\beta} \\
\left({ }^{H H} \nabla_{v^{v} X}\left({ }^{H H} \widetilde{Y}\right)\right)^{\beta}
\end{array}\right)
$$

are the components of $\left({ }^{H H} \nabla{ }^{v v} X\left({ }^{H H} \widetilde{Y}\right)\right)^{J}$ with respect to the coordinates $\left(x^{b}, x^{\beta}, x^{\bar{\beta}}\right)$ on $t\left(B_{m}\right)$, then we have

$$
\left({ }^{H H} \nabla_{{ }^{v} X}\left({ }^{H H} \widetilde{Y}\right)\right)^{J}={ }^{v v} X^{a H H} \nabla_{a}\left({ }^{H H} \widetilde{Y}\right)^{J}+{ }^{v v} X^{\alpha H H} \nabla_{\alpha}\left({ }^{H H} \widetilde{Y}\right)^{J}+{ }^{v v} X^{\bar{\alpha} H H} \nabla_{\bar{\alpha}}\left({ }^{H H} \widetilde{Y}\right)^{J}
$$

Firstly, if $J=b$, we have

$$
\begin{aligned}
\left({ }^{H H} \nabla_{{ }^{v v} X}\left({ }^{H H} \widetilde{Y}\right)\right)^{b} & =\underbrace{{ }^{v v} X^{a}}_{0}{ }^{H H} \nabla_{a}\left({ }^{H H} \widetilde{Y}\right)^{b}+\underbrace{{ }^{v v} X^{\alpha}}_{0}{ }^{H H} \nabla_{\alpha}\left({ }^{H H} \widetilde{Y}\right)^{b}+\underbrace{{ }^{v v} X^{\bar{\alpha}}}_{X^{\alpha}}{ }^{H H} \nabla_{\bar{\alpha}}\left({ }^{H H} \widetilde{Y}\right)^{b} \\
& \left.=X^{\alpha}(\underbrace{\partial_{\alpha} Y^{b}}_{0}+\underbrace{H H}_{0} \Gamma_{\bar{\alpha} c}^{b} c\left({ }^{H H} \widetilde{Y}\right)^{c}+\underbrace{{ }^{H H} \Gamma_{\bar{\alpha} \gamma}^{b}}_{0}\left({ }^{H H} \widetilde{Y}\right)^{\gamma}+\underbrace{{ }^{H H} \Gamma_{\bar{\alpha}}^{b}{ }^{H H}}_{0}{ }^{H H})^{\bar{\gamma}}\right) \\
& =0
\end{aligned}
$$


by virtue of (5), (9) and (16). Secondly, if $J=\beta$, we have

$$
\begin{aligned}
\left({ }^{H H} \nabla_{{ }^{v v} X}\left({ }^{H H} \widetilde{Y}\right)\right)^{\beta} & =\underbrace{{ }^{v v} X^{a}}_{0}{ }^{H H} \nabla_{a}\left({ }^{H H} \widetilde{Y}\right)^{\beta}+\underbrace{{ }^{v v} X^{\alpha}}_{0}{ }^{H H} \nabla_{\alpha}\left({ }^{H H} \widetilde{Y}\right)^{\beta}+\underbrace{{ }^{v} X^{\bar{\alpha}}}_{X^{\alpha}}{ }^{H H} \nabla_{\bar{\alpha}}\left({ }^{H H} \widetilde{Y}\right)^{\beta} \\
& =X^{\alpha}(\underbrace{\partial_{\alpha} Y^{\beta}}_{0}+\underbrace{{ }^{H H} \Gamma_{\bar{\alpha}}^{\beta} c}_{0}\left({ }^{H H} \widetilde{Y}\right)^{c}+\underbrace{{ }^{H H} \Gamma_{\bar{\alpha}}^{\beta} \gamma}_{0}\left({ }^{H H} \widetilde{Y}\right)^{\gamma}+\underbrace{{ }^{H H} \Gamma_{\bar{\alpha}}^{\beta} \bar{\gamma}}_{0}\left({ }^{H H} \widetilde{Y}\right)^{\bar{\gamma}}) \\
& =0
\end{aligned}
$$

by virtue of (5), (9) and (16). Thirdly, if $J=\bar{\beta}$, then we have

$$
\begin{aligned}
\left({ }^{H H} \nabla_{{ }^{v} X}\left({ }^{H H} \widetilde{Y}\right)\right)^{\bar{\beta}} & =\underbrace{{ }^{v} X^{a}}_{0}{ }^{H H} \nabla_{a}\left({ }^{H H} \widetilde{Y}\right)^{\bar{\beta}}+\underbrace{{ }^{v v} X^{\alpha}{ }^{H H}}_{0} \nabla_{\alpha}\left({ }^{H H} \widetilde{Y}\right)^{\bar{\beta}}+\underbrace{{ }^{v} X^{\bar{\alpha}}{ }^{H H} \nabla_{\bar{\alpha}}\left({ }^{H H} \widetilde{Y}\right)^{\bar{\beta}}}_{X^{\alpha}} \\
& =X^{\alpha}(-\underbrace{\partial_{\bar{\alpha}} y^{\varepsilon}}_{\delta_{\alpha}^{\varepsilon}} \Gamma_{\varepsilon}^{\beta} \gamma^{\gamma}+\underbrace{{ }^{H H} \Gamma_{\bar{\alpha}}^{\bar{\beta}}}_{0}\left({ }^{H H} \widetilde{Y}\right)^{c}+{ }^{H H} \Gamma_{\bar{\alpha}}^{\bar{\beta}} \gamma\left({ }^{H H} \widetilde{Y}\right)^{\gamma}+\underbrace{{ }^{H H} \Gamma_{\bar{\alpha}}^{\bar{\beta}} \bar{\gamma}}_{0}\left({ }^{H H} \widetilde{Y}\right)^{\bar{\gamma}}) \\
& =-X^{\alpha} \Gamma_{\alpha}^{\beta} Y^{\gamma}+X^{\alpha} \Gamma_{\alpha}^{\beta} Y^{\gamma} \\
& =0
\end{aligned}
$$

by virtue of (5), (9) and (16). The proof is completed.

Theorem 4. Let $\widetilde{X}$ and $\widetilde{Y}$ be projectable vector fields on $M_{n}$ with projection $X \in \mathfrak{I}_{0}^{1}\left(B_{m}\right)$ and $Y \in \mathfrak{I}_{0}^{1}\left(B_{m}\right)$, respectively. We have:

$$
{ }^{H H} \nabla_{c c} \widetilde{X}\left({ }^{H H} \widetilde{Y}\right)={ }^{H H}\left(\nabla_{X} Y\right) .
$$

Proof. (i) If $\widetilde{X}, \widetilde{Y} \in \mathfrak{I}_{0}^{1}\left(M_{n}\right)$ and

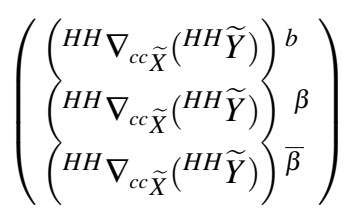

are the components of $\left({ }^{H H} \nabla_{c c}\left({ }^{H H} \widetilde{Y}\right)\right)^{J}$ with respect to the coordinates $\left(x^{b}, x^{\beta}, x^{\bar{\beta}}\right)$ on $t\left(B_{m}\right)$, then we have

$$
\left({ }^{H H} \nabla_{c c}\left({ }^{H H} \widetilde{Y}\right)\right)^{J}={ }^{c c} \widetilde{X}^{a H H} \nabla_{a}\left({ }^{H H} \widetilde{Y}\right)^{J}+{ }^{c c} \widetilde{X}^{\alpha H H} \nabla_{\alpha}\left({ }^{H H} \widetilde{Y}\right)^{J}+{ }^{c c} \widetilde{X}^{\bar{\alpha} H H} \nabla_{\bar{\alpha}}\left({ }^{H H} \widetilde{Y}\right)^{J}
$$

Firstly, if $J=b$, we have

$$
\begin{aligned}
\left({ }^{H H} \nabla_{c c}\left({ }^{H H} \widetilde{Y}\right)\right)^{b}= & { }^{c c} \widetilde{X}^{a H H} \nabla_{a}\left({ }^{H H} \widetilde{Y}\right)^{b}+{ }^{c c} \widetilde{X}^{\alpha H H} \nabla_{\alpha}\left({ }^{H H} \widetilde{Y}\right)^{b}+{ }^{c c} \widetilde{X}^{\bar{\alpha} H H} \nabla_{\bar{\alpha}}\left({ }^{H H} \widetilde{Y}\right)^{b} \\
= & X^{a H H} \nabla_{a}\left({ }^{H H} \widetilde{Y}\right)^{b}+X^{\alpha H H} \nabla_{a}\left({ }^{H H} \widetilde{Y}\right)^{b}+\left(y^{\varepsilon} \partial_{\varepsilon} X^{\alpha}\right)^{H H} \nabla_{a}\left({ }^{H H} \widetilde{Y}\right)^{b} \\
= & X^{a}(\partial_{a} Y^{b}+\underbrace{{ }^{H H} \Gamma_{a}^{b} c}_{0} Y^{c}+\underbrace{{ }^{H H} \Gamma_{a}^{b} \gamma}_{0} Y^{\gamma}+\underbrace{{ }^{H H} \Gamma_{a}^{b} Y^{\gamma}}_{0} Y^{\bar{\gamma}})+X^{\alpha}(\partial_{\alpha} Y^{b}+\underbrace{H H}_{0} \Gamma_{\alpha c}^{b} Y^{c}+\underbrace{{ }^{H H} \Gamma_{\alpha}^{b}}_{\Gamma_{\alpha}^{b}} Y^{\gamma}+\underbrace{H H}_{0} \Gamma_{\alpha \bar{\gamma}}^{b} Y^{\bar{\gamma}}) \\
& +\left(y^{\varepsilon} \partial_{\varepsilon} X^{\alpha}\right)(\underbrace{\partial_{\alpha} Y^{b}}_{0}+\underbrace{{ }^{H H} \Gamma_{\alpha}^{b} c}_{0} Y^{c}+\underbrace{{ }^{H H} \Gamma_{\alpha}^{b} \gamma}_{0} Y^{\gamma}+\underbrace{{ }^{H H} \Gamma_{\alpha}^{b} \bar{\gamma}}_{0} Y^{\bar{\gamma}}) \\
= & X^{\alpha} \partial_{\alpha} Y^{b}+X^{\alpha} \Gamma_{\alpha}^{b} Y^{\gamma}=X^{\alpha}\left(\partial_{\alpha} Y^{b}+\Gamma_{\alpha}^{b} Y^{\gamma}\right)
\end{aligned}
$$


by virtue of (7), (9) and (16). Secondly, if $J=\beta$, we have

$$
\begin{aligned}
& \left({ }^{H H} \nabla_{c c}\left({ }^{H H} \widetilde{Y}\right)\right)^{\beta}={ }^{c c} \widetilde{X}^{a H H} \nabla_{a}\left({ }^{H H} \widetilde{Y}\right)^{\beta}+{ }^{c c} \widetilde{X}^{\alpha H H} \nabla_{\alpha}\left({ }^{H H} \widetilde{Y}\right)^{\beta}+{ }^{c c} \widetilde{X}^{\bar{\alpha} H H} \nabla_{\bar{\alpha}}\left({ }^{H H} \widetilde{Y}\right)^{\beta} \\
& =X^{a H H} \nabla_{a}\left({ }^{H H} \widetilde{Y}\right)^{\beta}+X^{\alpha H H} \nabla_{\alpha}\left({ }^{H H} \widetilde{Y}\right)^{\beta}+\left(y^{\varepsilon} \partial_{\varepsilon} X^{\alpha}\right)^{H H} \nabla_{\bar{\alpha}}\left({ }^{H H} \widetilde{Y}\right)^{\beta} \\
& =X^{a}(\underbrace{\partial_{a} Y^{\beta}}_{0}+\underbrace{{ }^{H H} \Gamma_{a}^{\beta} c}_{0} Y^{c}+\underbrace{{ }^{H H} \Gamma_{a}^{\beta} \gamma}_{0} Y^{\gamma}+\underbrace{{ }^{H H} \Gamma_{a}^{\beta} \bar{\gamma}}_{0} Y^{\bar{\gamma}})+X^{\alpha}(\partial_{\alpha} Y^{\beta}+\underbrace{{ }^{H H} \Gamma_{\alpha c}^{\beta} Y^{c}}_{0}+\underbrace{\beta}_{\Gamma_{\alpha \gamma}^{\beta}{ }^{H H} \Gamma_{\alpha}^{\beta}} Y^{\gamma}+\underbrace{{ }^{H H} \Gamma_{\alpha}^{\beta}}_{0} Y^{\bar{\gamma}}) \\
& +\left(y^{\varepsilon} \partial_{\varepsilon} X^{\alpha}\right)(\underbrace{\partial_{\bar{\alpha}} Y^{\beta}}_{0}+\underbrace{{ }^{H H} \Gamma_{\bar{\alpha}}^{\beta} c}_{0} Y^{c}+\underbrace{{ }^{H H} \Gamma_{\bar{\alpha}}^{\beta} \gamma}_{0} Y^{\gamma}+\underbrace{{ }^{H H} \Gamma_{\bar{\alpha}}^{\beta} \bar{\gamma}}_{0} Y^{\bar{\gamma}}) \\
& =X^{\alpha} \partial_{\alpha} Y^{\beta}+X^{\alpha} \Gamma_{\alpha \gamma}^{\beta} Y^{\gamma}=X^{\alpha}\left(\partial_{\alpha} Y^{\beta}+\Gamma_{\alpha}^{\beta}{ }^{\beta}{ }^{\gamma}\right)
\end{aligned}
$$

by virtue of (7), (9) and (16). Thirdly, if $J=\bar{\beta}$, then we have

$$
\begin{aligned}
\left({ }^{H H} \nabla_{c c}^{H H} \widetilde{Y}\right)^{\bar{\beta}}= & { }^{c} \widetilde{X}^{a H H} \nabla_{a}\left({ }^{H H} \widetilde{Y}\right)^{\bar{\beta}}+{ }^{c c} \widetilde{X}^{\alpha H H} \nabla_{\alpha}\left({ }^{H H} \widetilde{Y}\right)^{\bar{\beta}}+{ }^{c c} \widetilde{X}^{\bar{\alpha} H H} \nabla_{\bar{\alpha}}\left({ }^{H H} \widetilde{Y}\right)^{\bar{\beta}} \\
= & X^{a H H} \nabla_{a}\left(-y^{\varepsilon} \Gamma_{\varepsilon}^{\beta}{ }_{\sigma} Y^{\sigma}\right)+X^{\alpha H H} \nabla_{\alpha}\left(-y^{\varepsilon} \Gamma_{\varepsilon}^{\beta}{ }_{\sigma} Y^{\sigma}\right)+\left(y^{\varepsilon} \partial_{\varepsilon} X^{\alpha}\right)^{H H} \nabla_{\bar{\alpha}}\left(-y^{\varepsilon} \Gamma_{\varepsilon}^{\beta}{ }_{\sigma} Y^{\sigma}\right) \\
= & -X^{a} \underbrace{\partial_{a} \Gamma_{\varepsilon}^{\beta} \sigma}_{0} y^{\varepsilon} Y^{\sigma}-X^{a} \underbrace{\partial_{a} y^{\varepsilon}}_{0} \Gamma_{\varepsilon}^{\beta}{ }_{\sigma} Y^{\sigma}-X^{a} \Gamma_{\varepsilon}^{\beta} \sigma y^{\varepsilon} \underbrace{\partial_{a} Y^{\sigma}}_{0}-X^{\alpha} \partial_{\alpha} \Gamma_{\varepsilon}^{\beta} \sigma y^{\varepsilon} Y^{\sigma} \\
& -X^{\alpha} \underbrace{\partial_{\alpha} y^{\varepsilon}}_{0} \Gamma_{\varepsilon}^{\beta}{ }_{\sigma} Y^{\sigma}-X^{\alpha} \Gamma_{\varepsilon}^{\beta} \sigma y^{\varepsilon} \partial_{\alpha} Y^{\sigma}+X^{\alpha} y^{\varepsilon} \partial_{\varepsilon} \Gamma_{\alpha}^{\beta}{ }_{\sigma} Y^{\sigma}-X^{\alpha} y^{\varphi} \partial_{\varphi} \Gamma_{\alpha}^{\beta}{ }_{\sigma} Y^{\sigma}+X^{\alpha} y^{\varphi} \partial_{\alpha} \Gamma_{\varphi}^{\beta}{ }_{\sigma} Y^{\sigma} \\
& -X^{\alpha} y^{\varphi} \Gamma_{\varphi}^{\beta}{ }_{\phi} \Gamma_{\alpha}^{\phi}{ }^{\phi} Y^{\sigma}+X^{\alpha} y^{\varphi} \Gamma_{\alpha}^{\beta}{ }_{\phi} \Gamma_{\varphi}^{\phi} \sigma Y^{\sigma}-X^{\alpha} \Gamma_{\alpha}^{\beta} \sigma \Gamma_{\varepsilon}^{\sigma}{ }_{\phi} y^{\varepsilon} Y^{\phi}-\Gamma_{\varepsilon}^{\beta} \sigma y^{\varepsilon} X^{\alpha} \partial_{\alpha} Y^{\sigma}+\Gamma_{\varepsilon}^{\beta} \sigma y^{\varepsilon} X^{\alpha} \partial_{\alpha} Y^{\sigma} \\
= & -\Gamma_{\varepsilon}^{\beta} \sigma y^{\varepsilon} X^{\alpha} \partial_{\alpha} Y^{\sigma}+\Gamma_{\varphi}^{\beta}{ }_{\phi} \Gamma_{\alpha}^{\phi} \sigma X^{\alpha} y^{\varphi} Y^{\sigma}
\end{aligned}
$$

by virtue of (7), (9) and (16). Thus, we have ${ }^{H H} \nabla_{c c}\left({ }^{H H} \widetilde{Y}\right)={ }^{H H}\left(\nabla_{X} Y\right)$.

Theorem 5. Let $\widetilde{X}$ be a projectable vector field on $M_{n}$ with projections $X$ on $B_{m}$. If $Y \in \mathfrak{I}_{0}^{1}\left(B_{m}\right)$, then

$$
{ }^{H H} \nabla_{H H \tilde{X}}\left({ }^{v v} Y\right)={ }^{v v}\left(\nabla_{X} Y\right)
$$

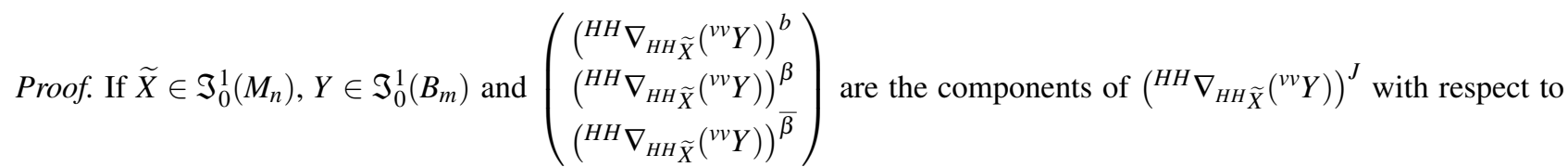
the coordinates $\left(x^{b}, x^{\beta}, x^{\bar{\beta}}\right)$ on $t\left(B_{m}\right)$, then we have

$$
\left({ }^{H H} \nabla_{H H \widetilde{X}}\left({ }^{v v} Y\right)\right)^{J}={ }^{H H} \widetilde{X}^{a H H} \nabla_{a}\left({ }^{v v} Y\right)^{J}+{ }^{H H} \widetilde{X}^{\alpha H H} \nabla_{\alpha}\left({ }^{v v} Y\right)^{J}+{ }^{H H} \widetilde{X}^{\bar{\alpha} H H} \nabla_{\bar{\alpha}}\left({ }^{v v} Y\right)^{J} .
$$

Firstly, if $J=b$, we have

$$
\begin{aligned}
& \left({ }^{H H} \nabla_{H H} \widetilde{X}\left({ }^{v v} Y\right)\right)^{b}={ }^{H H} \widetilde{X}^{a H H} \nabla_{a}\left({ }^{v v} Y\right)^{b}+{ }^{H H} \widetilde{X}^{\alpha H H} \nabla_{\alpha}\left({ }^{v v} Y\right)^{b}+{ }^{H H} \widetilde{X}^{\bar{\alpha} H H} \nabla_{\bar{\alpha}}\left({ }^{v v} Y\right)^{b} \\
& =X^{a}(\partial_{a} \underbrace{{ }^{v v} Y^{b}}_{0}+{ }^{H H} \Gamma_{a}^{b}{ }^{b v} \underbrace{v v}_{0} Y^{c}+{ }^{H H} \Gamma_{a}^{b} \gamma_{0}^{v^{v v} Y^{\gamma}}+\underbrace{{ }^{H H} \Gamma_{a}^{b} \bar{\gamma}}_{0}\left({ }^{v v} Y\right)^{\bar{\gamma}})
\end{aligned}
$$

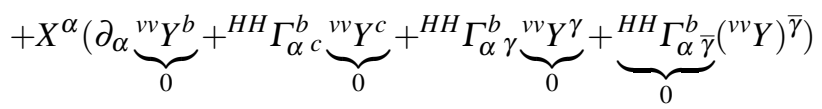

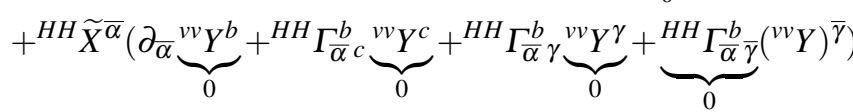

$$
\begin{aligned}
& =0
\end{aligned}
$$


by virtue of (5), (9) and (16). Secondly, if $J=\beta$, we have

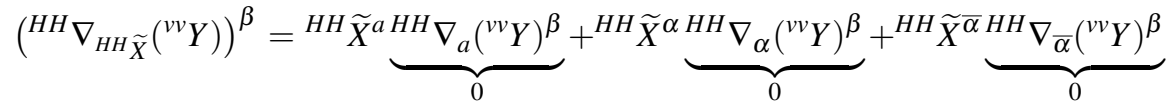

$$
\begin{aligned}
& \left.=X^{a}(\partial_{a} \underbrace{{ }^{v v} Y^{\beta}}_{0}+{ }^{H H} \Gamma_{a}^{\beta} c_{0}^{{ }^{v v} Y^{c}}+{ }^{H H} \Gamma_{a}^{\beta} \gamma_{0}^{\gamma^{v v} Y^{\gamma}}+\underbrace{H H}_{0} \Gamma_{a}^{\beta} \bar{\gamma}^{\left({ }^{v v} Y\right.})^{\bar{\gamma}}\right) \\
& \left.+X^{\alpha}(\partial_{\alpha} \underbrace{v^{v} Y^{\beta}}_{0}+{ }^{H H} \Gamma_{\alpha}^{\beta} c \underbrace{{ }^{v v} Y^{c}}_{0}+{ }^{H H} \Gamma_{\alpha}^{\beta} \gamma_{0}^{{ }^{v v} Y^{\gamma}}+\underbrace{H H}_{0} \Gamma_{\alpha}^{\beta} \bar{\gamma}^{(v v} Y)^{\bar{\gamma}}\right) \\
& +{ }^{H H} \widetilde{X}^{\bar{\alpha}}(\partial_{\bar{\alpha}} \underbrace{v^{v} Y^{\beta}}_{0}+{ }^{H H} \Gamma_{\bar{\alpha}}^{\beta} c \underbrace{{ }^{v v} Y^{c}}_{0}+{ }^{H H} \Gamma_{\bar{\alpha}}^{\beta} \gamma_{0}^{{ }^{v v} Y^{\gamma}}+\underbrace{H H}_{0} \Gamma_{\bar{\alpha}}^{\beta} \bar{\gamma}\left({ }^{(v v} Y\right)^{\bar{\gamma}}) \\
& =0
\end{aligned}
$$

by virtue of (5), (9) and (16). Thirdly, if $J=\bar{\beta}$, then we have

$$
\begin{aligned}
& \left.\left({ }^{H H} \nabla_{H H} \widetilde{X}^{v v} Y\right)\right)^{\bar{\beta}}={ }^{H H} \widetilde{X}^{a H H} \nabla_{a} \underbrace{{ }^{v v} Y^{\bar{\beta}}}_{0}+{ }^{H H} \widetilde{X}^{\alpha H H} \nabla_{\alpha} \underbrace{{ }^{v v} Y^{\bar{\beta}}}_{0}+{ }^{H H} \widetilde{X}^{\bar{\alpha} H H} \nabla_{\bar{\alpha}} \underbrace{{ }^{v} Y^{\bar{\beta}}}_{0} \\
& =X^{a}(\underbrace{\partial_{a}\left({ }^{v v} Y\right)^{\bar{\beta}}}_{0}+{ }^{H H} \Gamma_{a}^{\bar{\beta}} c\left({ }^{(v v} Y\right)^{c}+\underbrace{H H}_{0} \Gamma_{a}^{\bar{\beta}} \gamma\left({ }^{v v} Y\right)^{\gamma}+\underbrace{H H}_{0} \Gamma_{a}^{\bar{\beta}} \bar{\gamma}\left({ }^{(v v} Y\right)^{\bar{\gamma}}) \\
& \left.+X^{\alpha}(\partial_{\alpha}\left({ }^{v v} Y\right)^{\bar{\beta}}+{ }^{H H} \Gamma_{\alpha}^{\bar{\beta}} c\left({ }^{\left({ }^{v} V\right.} Y\right)^{c}+{ }^{H H} \Gamma_{\alpha}^{\bar{\beta}} \gamma \underbrace{\left({ }^{v v} Y\right)^{\gamma}}_{0}+\underbrace{H H}_{\Gamma_{\alpha}^{\beta} \gamma} \Gamma_{\alpha}^{\bar{\beta}} \bar{\gamma}^{\left({ }^{\beta} v\right.} Y)^{\bar{\gamma}}\right) \\
& +\left(-y^{\varepsilon} \Gamma_{\varepsilon}^{\alpha} \beta Y^{\beta}\right)(\underbrace{\partial_{\alpha} Y^{\beta}}_{0}+{ }^{H H} \Gamma_{\bar{\alpha}}^{\bar{\beta}} \underbrace{v^{v} Y^{c}}_{0}+{ }^{H H} \Gamma_{\bar{\alpha}}^{\bar{\beta}} \gamma_{0}^{{ }^{v v} Y^{\gamma}}+\underbrace{{ }^{H H} \Gamma_{\bar{\alpha}}^{\bar{\beta}} \bar{\gamma}}_{0}\left({ }^{v v} Y\right)^{\bar{\gamma}}) \\
& =X^{\alpha} \partial_{\alpha} Y^{\beta}+X^{\alpha} \Gamma_{\alpha \gamma}^{\beta} Y^{\gamma}=X^{\alpha}\left(\partial_{\alpha} Y^{\beta}+\Gamma_{\alpha \gamma}^{\beta} Y^{\gamma}\right) \\
& =\left(\nabla_{X} Y\right)^{\beta}
\end{aligned}
$$

by virtue of (5), (9) and (16). On the other hand, we know that ${ }^{v v}\left(\nabla_{X} Y\right)$ have the components

$$
{ }^{v v}\left(\nabla_{X} Y\right)=\left(\begin{array}{l}
0 \\
0 \\
\left(\nabla_{X} Y\right)^{\beta}
\end{array}\right)
$$

with respect to the coordinates $\left(x^{b}, x^{\beta}, x^{\bar{\beta}}\right)$ on $t\left(B_{m}\right)$. Thus, we have ${ }^{H H} \nabla_{H H \tilde{X}}\left({ }^{v v} Y\right)={ }^{v v}\left(\nabla_{X} Y\right)$ in $t\left(B_{m}\right)$.

\section{Conclusion}

In this paper, we consider horizontal lifting problem of projectable linear connection on $\mathrm{M}$ to the semi-tangent bundle tM. In this context, the following equations have been obtained:

(i) ${ }^{H H} \nabla^{{ }^{v}} X\left({ }^{v v} Y\right)=0$,

(ii) ${ }^{H H} \nabla_{v^{v} X}\left({ }^{H H} \widetilde{Y}\right)=0$,

(iii) ${ }^{H H} \nabla_{c c}\left({ }^{H H} \widetilde{Y}\right)={ }^{H H}\left(\nabla_{X} Y\right)$,

(iv) ${ }^{H H} \nabla_{H H \tilde{X}}\left({ }^{v v} Y\right)={ }^{v v}\left(\nabla_{X} Y\right)$. 


\section{Competing interests}

The authors declare that they have no competing interests.

\section{Authors' contributions}

All authors have contributed to all parts of the article. All authors read and approved the final manuscript.

\section{Acknowledgment}

The paper was supported by TUBITAK project MFAG-118F176.

\section{References}

[1] A. Bednarska, On lifts of projectable-projectable classical linear connections to the cotangent bundle, Annales Universitatis Mariae Curie-Skłodowska, Sectio A, Mathematica, 67 (2013), no. 1, 1-10.

[2] D. Husemoller, Fibre Bundles. Springer, New York, 1994.

[3] H.B. Lawson and M.L. Michelsohn, Spin Geometry. Princeton University Press., Princeton, 1989.

[4] W.M. Mikulski and J. Tomáš, Reduction for natural operators on projectable connections, Demonstratio Mathematica; 42 (2009), no. 2, 437-441.

[5] Mikulski, W.M., On the existence of prolongations of connections by bundle functors, Extracta Math. 22 (2007), no. 3, $297-314$.

[6] N.M. Ostianu, Step-fibred spaces, Tr. Geom. Sem. 5, Moscow. (VINITI), (1974), 259-309.

[7] L.S. Pontryagin, Characteristic cycles on differentiable manifolds. Amer. Math. Soc. Translation, (1950), no. 32, 72 pp.

[8] W.A. Poor, Differential Geometric Structures, New York, McGraw-Hill, 1981.

[9] A.A. Salimov and E. Kadığlu, Lifts of Derivations to the Semitangent Bundle, Turk J. Math. 24 (2000), 259-266.

[10] N. Steenrod, The Topology of Fibre Bundles. Princeton University Press., Princeton, 1951.

[11] V.V. Vishnevskii, Integrable affinor structures and their plural interpretations. Geometry, 7.J. Math. Sci. (New York) 108 (2002), no. $2,151-187$.

[12] V.V. Vishnevskii, A.P. Shirokov and V.V. Shurygin, Spaces over Algebras. Kazan. Kazan Gos. Univ. 1985. (in Russian).

[13] K. Yano and S. Ishihara, Tangent and Cotangent Bundles. Marcel Dekker, Inc., New York, 1973.

[14] F. Y1ldırım and A. Salimov, Semi-cotangent bundle and problems of lifts, Turk J. Math, 38 (2014), 325-339.

[15] F. Yıldırım, On a special class of semi-cotangent bundle, Proceedings of the Institute of Mathematics and Mechanics, (ANAS) 41 (2015), no. 1, 25-38. 